\title{
La dialisi peritoneale in Italia nel 2000. I perché di una penetrazione nel trattamento dell'uremia ferma da anni al $10 \%$
}

\author{
Renzo Scanziani
}

Servizio di Nefrologia e Dialisi, Ospedale di Desio, Desio, Milano

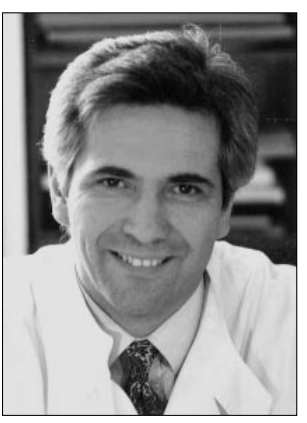

Diversi fattori sia oggettivi che soggettivi concorrono a fare in modo che la dialisi peritoneale (DP) in Italia e non solo, venga ancora considerata una dialisi "marginale", pur essendo una metodica non più "giovanissima".

Le controindicazioni assolute e relative alle diverse tecniche depurative giocano tuttora un ruolo essenziale nella scelta del tipo di trattamento, nonostante il progressivo miglioramento della tecnologia e delle conoscenze della DP.

Mentre l'unica controindicazione assoluta all'emodialisi (ED) è l'impossibilità di disporre di un valido accesso vascolare e le relative sono la severa instabilità emodinamica e $\mathrm{i}$ gravi disordini della coagulazione, le controindicazioni assolute alla DP vanno dall'impossibilità a utilizzare la membrana peritoneale per il trattamento dialitico (estese aderenze peritoneali per pregressi interventi addominali e/o problemi meccanici addominali non correggibili chirurgicamente) all'indisponibilità fisica o cognitiva (anche per l'assenza di part- ner) per l'esecuzione pratica della tecnica. Le controindicazioni relative vanno dalle patologie della cavità o della parete addominale (infettive e/o infiammatorie o ischemiche) all'impossibilità a garantire un adeguato trattamento dialitico (pazienti di grossa taglia corporea, obesità patologica, intolleranza a volumi adeguati di dialisato, severa malnutrizione). È evidente che questa grande disparità ha fin dall'inizio un ruolo determinante nell'indicazione alla tecnica dialitica, disparità che negli ultimi anni è andata sicuramente aumentando sia per l'invecchiamento della popolazione avviata a terapia sostitutiva sia per una generale minore disponibilità (con o senza partner) alla pratica domiciliare, che già negli anni '80-'90 ha prodotto il declino della ED domiciliare. La minor disponibilità al trattamento domiciliare ha anche determinato il passaggio di pazienti dal trattamento DP manuale (CAPD) a quello automatizzato (APD), che si è osservato negli ultimi anni in Italia e nel mondo.

Il ruolo del nefrologo risulta fondamentale nella scelta della tecnica dialitica come dimostra l'alta percentuale di pazienti avviati alla DP in alcuni Centri sia italiani che europei indipendentemente dal fatto che siano "early" o "late referral".

Le sue conoscenze sono in grado da un lato di restringere o ampliare le controindicazioni relative alla DP, dall'altro di garantire un adeguato utilizzo delle diverse modalità di DP (CAPD o APD) sia in funzione delle necessità cliniche (adeguatezza dialitica, complicanze meccaniche addominali) sia sociali (gestione dei tempi, necessità o meno di partner) dei pazienti. Indipendentemente dal "fattore Centro", e nonostante i miglioramenti tecnologici (connettologia, materiali e soluzioni con migliore biocompatibilità) e le sempre più ampie conoscenze, le complicanze infettive o non proprie della metodica e $\mathrm{i}$ problemi legati allo "stato biologico" della membrana peritoneale (idonea e persistente permeabilità peritoneale ai soluti per le necessità di adeguatezza dialitica) restano a tutt'oggi fattori determinanti del drop out e limitanti la sopravvivenza della metodica nel lungo termine soprattutto dopo la perdita della funzione renale residua (FRR).

$\mathrm{E}$ indubbio che in questi anni altri fattori hanno condizionato la scelta del tipo di trattamento dell'uremia da parte del nefrologo.

Se è vero che solo grazie ai notevoli investimenti industriali le sempre più ampie conoscenze mediche sulla DP hanno permesso di realizzare rapidamente quei miglioramenti tecnologici necessari a una sua ampia afferma- 
zione, è altrettanto vero che un ruolo determinante alla sua diffusione è stato il tipo di assistenza sanitaria (pubblica o privata, o una presenza paritetica tra assistenza pubblica e privata) presente nei vari Paesi e da cui sono derivati i diversi modelli organizzativi utilizzati per il trattamento dell'uremia.

L'intrecciarsi di questi diversi interessi (scientifici, industriali anche nazionali, economici sia privati che pubblici) ha poi portato ad atti legislativi e interventi finanziari che hanno determinato la notevole diversa penetrazione della DP presente oggi nei diversi Paesi. In alcuni Paesi hanno sicuramente pesato specifici interventi legislativi, come la Francia, in cui vi sono stati una limitazione dei centri ED e un libero accesso alla $\mathrm{DP}$, in altri Paesi hanno pesato gli interventi economici, come la Gran Bretagna che ha concesso scarsi finanziamenti alla ED. È interessante constatare come l'attuale differente diffusione nel mondo della DP veda come elemento dirimente il rapporto tra intervento pubblico e privato nel trattamento dell'uremia; di norma nei Paesi in cui maggiore è la presenza dell'intervento privato minore è l'utilizzo della DP.

\section{In Italia che cosa è successo?}

Al momento della comparsa della CAPD in Italia il trattamento dell'uremia prevedeva l'intervento del pubblico e del privato convenzionato il cui rapporto era diversificato da $\mathrm{Re}$ gione a Regione, in un sistema di finanziamento dell' assistenza basato su una spesa prevista e poi ripianata a consuntivo. Così negli anni '80 la DP si diffonde in maniera differente da Regione a Regione. Dove è storicamente maggiore la presenza dell' iniziativa privata nel trattamento dell'uremia la DP stenta ad affermarsi mentre dove questa è minoritaria o assente la DP facilmente si insedia e si espande. Inoltre la maggior morbilità della DP nei primi anni della sua introduzione ha fatto sì che giuste preoccupazioni di ordine clinico producessero un impianto legislativo che ha di fatto impedito l'effettuazione della DP nelle strutture private.
A metà degli anni '90 di fronte alla crescita esponenziale della spesa sanitaria si avverte la necessità di introdurre correttivi nei finanziamenti dell'assistenza che tengano conto anche delle leggi di mercato.

Si ha così in Italia l'introduzione di un sistema con una visione aziendale della Sanità, in cui la spesa complessiva rimane limitata nell'ambito di un finanziamento predefinito e non ripianabile, se non per autofinanziamento regionale, e basato sul rimborso di singole prestazioni secondo una tariffa predefinita.

In realtà tale condizione non rappresenta una condizione di mercato poiché si basa su una tariffa unica per qualsiasi livello di prestazione (purché in possesso di un livello definito di requisito minimo) e, di fatto, non permette una reale concorrenza tra pubblico e privato che presentano diversità operativa (modalità di acquisto di beni, servizi e personale e di accesso al credito), profonda differenza nella possibilità e diritto di selezione (obbligo del pubblico all'assistenza) con l'impossibilità della libera scelta dell'utente. Tale scelta sarebbe possibile solo attraverso una qualificazione dei livelli di prestazione e quindi delle strutture attraverso tariffe differenziate.

Per quanto riguarda il trattamento dell'uremia succede che la quantificazione dei costi delle differenti prestazioni risente della convinzione diffusa di una particolare economicità intrinseca della DP, non sempre vero nella pratica clinica (anche se mediamente il costo globale della DP è inferiore a quello della ED), e del fatto che essa presenta vantaggi economici specifici nei confronti della ED non adeguatamente presi in considerazione nella valutazione dei rimborsi (minore necessità strutturale con i relativi risparmi impiantistici, forte riduzione di fabbisogno del personale, netta riduzione delle spese di accompagnamento). A questo vanno aggiunti le evidenti più benevoli valutazioni economiche verso la ED delle quali sono una spia significativa le diverse tariffe per i costi dei trattamenti ED a fronte delle due sole tariffe (CAPD o
APD) previste per la DP.

Il confronto fra costi dei trattamenti dialitici e tariffe ministeriali di rimborso dimostra che a fronte di una generale sottovalutazione media dei costi reali dei vari trattamenti la dialisi peritoneale è maggiormente sottostimata, soprattutto la APD, rispetto alla ED. Questa sottostima è rimasta anche dopo le modifiche tariffarie introdotte in ambito regionale che pur mostrano valutazioni differenziate dei costi e quindi dei rimborsi. Ciò costituisce una condizione negativa non solo per una maggiore estensione della DP, ma anche per un suo mantenimento agli attuali livelli di penetrazione.

E sicuramente significativo constatare che nonostante queste modifiche la situazione della diffusione attuale della DP in ambito regionale rispecchia la differente penetrazione delle metodiche precedentemente presenti. Il mancato riequilibrio economico fra $\mathrm{DP}$ e ED, con il persistere di una condizione di rimborso assai favorevole alla $\mathrm{ED}$, ha attirato ed attirerà in modo crescente l'attenzione dell'imprenditoria privata specifica con investimenti diretti nell'assistenza solo in una direzione e nulla verrà fatto per rimuovere quelle carenze anche legislative che permetterebbero una corretta concorrenza tra pubblico e privato.

Senza un reale riequilibrio tra i costi e i relativi rimborsi la DP rimarrà priva degli incentivi necessari a un suo libero e pieno utilizzo nell'attuale visione aziendale della sanità. Verranno così a mancare i finanziamenti e la spinta industriale alla ricerca e la dialisi peritoneale avrà sempre più un ruolo di terapia "marginale".

juscanz@tin.it 